\title{
Feeling Smooth: Psychotribological Probing of Molecular Composition
}

\author{
Lisa Skedung ${ }^{1} \cdot$ Kathryn Harris $^{1}$ - Elizabeth S. Collier ${ }^{1} \cdot$ Martin Arvidsson $^{1,4}$ - Aneliia Wäckerlin ${ }^{2}$. Walter Haag ${ }^{2}$. \\ Marco Bieri $^{2,5} \cdot$ Andriy Romanyuk $^{2} \cdot$ Mark W. Rutland $^{1,3}$
}

Received: 27 November 2017 / Accepted: 20 August 2018 / Published online: 27 September 2018

(c) The Author(s) 2018

\begin{abstract}
The aim of this study was to evaluate whether smooth surfaces varying in surface chemistry could be perceptually distinguished with the sense of touch. A set of ten glass surfaces was prepared which varied systematically in terms of the molecular composition of a thin coating of low topography. The contact angle, contact angle hysteresis, and surface energy were evaluated as objective physical parameters characterizing each coating. Additionally, the interaction forces between a human finger and the different coatings were quantified and compared in terms of tactile friction coefficients. The surfaces were evaluated psychophysically in terms of perceived similarities and were then ranked according to pleasantness. The participants could perceptually distinguish between surfaces varying in surface chemistry and a primary and secondary perceptual dimension were identified as sufficient to distinguish them. The primary dimension correlates with surface free energy, but both tactile friction and surface energy contribute to this dimension depending on whether the coatings are organic or inorganic. The secondary dimension could not be identified explicitly in terms of a physical quantity but is discussed in terms of recent developments in the literature. Coated glass is characterized by high friction coefficient upon interaction with a human finger as well as significant hysteresis in the stroking directions (lower applied load and higher friction in the backward stroke). Despite the complexity of the tribology, pleasantness can be clearly linked to it, where low friction (high contact angle) materials receive a higher ranking.
\end{abstract}

Keywords Tactile perception · Tactile discrimination $\cdot$ Surface chemistry $\cdot$ Psychotribology $\cdot$ Biotribology $\cdot$ Index finger . Tactile friction . Active touch

Electronic supplementary material The online version of this article (https://doi.org/10.1007/s11249-018-1077-z) contains supplementary material, which is available to authorized users.

Mark W. Rutland

mark@kth.se

1 Bioscience and Materials, RISE Research Institutes of Sweden, 11428 Stockholm, Sweden

2 Glas Trösch AG, Industriestrasse 29, 4922 Bützberg, Switzerland

3 Surface and Corrosion Science, KTH Royal Institute of Technology, 10044 Stockholm, Sweden

4 Present Address: Tobii AB (publ), Box 743, 18217 Danderyd, Sweden

5 Present Address: Swiss National Science Foundation, Wildhainweg 3, 3001 Berne, Switzerland

\section{Introduction}

Tactile perception of fine textures and the role of topography and other physical parameters is currently receiving considerable attention [1-3], as materials science developments allow single parameters to be varied systematically across a series of surfaces. For example, nanometre scale amplitude on patterned surfaces can be distinguished from smooth surfaces [1]. However, the extent to which the chemistry of the surface can be detected using active tactile exploration is not known. Probing this parameter requires the manufacture of a series of surfaces where the roughness is below the detection limit but where the chemical constituents vary, for example, from polar to non-polar. Glass can be manufactured to be very smooth, and industrial glass coating technology is capable of forming smooth, homogeneous films of thickness in the range $1-2 \mathrm{~nm}$ with contact angles against water varying from $0^{\circ}$ to approximately $120^{\circ}$. The smoothness of the glass and its use in a myriad of applications coupled with 
the ease of such chemical modification means that it is an ideal substrate to isolate the surface chemistry as a parameter. Tactile friction of glass has been studied as a substrate before for example by Derler et al. [4] who looked at the effect of roughness and were able to show that the adhesive friction was sufficient to explain the observed trends. More recently, Adams et al. [5] have reviewed the role of fingerpad friction in touch. They also addressed the contact mechanics, this time from the perspective of the fingerprint ridges, and identified the role of sweat and the occlusion of moisture [6], which is particularly important for non-porous, smooth surfaces such as glass and leads to an increased friction in that case. They also identified friction as being important for perceived pleasantness. Geuorguiev et al. [7] reported on friction as a cue in touching flat surfaces, and suggested that discrimination ability was facilitated by the dynamics preceding steady sliding. The surfaces nonetheless displayed features on the scale of $10 \mathrm{~s}$ of $\mathrm{nm}$, a scale where topographic effects on the true area of contact can lead to perceptual differences [1].

It is known from the literature that roughness-smoothness, hardness-softness, and stickiness-slipperiness are predominant perceptual dimensions in macro texture perception [8]. Previous work has shown that the perceptual dimensionality is intact when reducing the texture scale down to microns, but that the differences between the dimensions start to blur [2]. It is possible to demonstrate that the sticky-slippery dimension can be correlated with perceived pleasantness [9]. It has also been shown through recent neurological work that the mechanoreceptors of the hand overlap to a greater extent in the perception of micro textures than for larger structures where temporal and spatial cues trigger different receptors. It is thus unclear which perceptual dimensions will govern perception on smooth surfaces (roughness amplitude below $6 \mathrm{~nm}$ ) [1], and which surface properties influence the feel of such surfaces.

In the present work, a similarity scaling experiment to determine which tactile factors are important in "molecular touch" has been performed. In a complementary set of tests, friction coefficients have been determined for the interaction with a finger. Each surface was additionally characterized by water contact angle (WCA) measurement, and by morphology/roughness using atomic force microscopy (AFM). Correlations between the tactile perception and the surface chemistry are discussed.

\section{Experimental}

\subsection{Materials}

In order to explore the surface chemical tactile space, we prepared a series of surfaces with similarly low roughness, where the chemical constituents vary systematically from polar to non-polar, and the water contact angles of the surfaces vary from $0^{\circ}$ to approximately $120^{\circ}$.

Float glass surface was taken as a smooth substrate. The smooth homogeneous thin films $(<10 \mathrm{~nm})$ were deposited on clean glass. In total we prepared 1500 samples: 150 samples of each of 10 coating types. The 1500 samples were sufficient to conduct all physical measurements and to present a fresh sample for each comparison in the perception studies without the need to develop cleaning procedures. The samples used in the experiments are presented in Table 1. There are three classes of films: \#1-4 inorganic, \#6-8 hybrid (organic/inorganic), \#9-10 organic.

\subsection{Tactile Friction Measurements}

Finger friction or tactile friction was measured with a ForceBoard $^{\mathrm{TM}}$ (Industrial Dynamics AB, Sweden), which is equipped with two load cells, one horizontal and one tangential, consisting of strain gauges in a Wheatstone bridge configuration. A mechanical load is converted into voltage signals that are amplified and proportional to the applied load. The tangential force, i.e., friction force, and vertical force, i.e., applied load, were continuously recorded using DAQFactory software at a rate of $100 \mathrm{~Hz}$ as a finger was moved over the glass surface. The output data were a text file of columns with the time and respective forces and the analysis was performed using MATLAB.

The glass surfaces were mounted on the top plate with double-sided adhesive tape, and one female experimenter probed the surface with her dominant index finger with a finger sliding angle of approximately $30^{\circ}$. The sliding direction was along the finger axis for all tests. The average stroking distance was about $80 \mathrm{~mm}$ and the average speed of all measurements was approximately $20 \mathrm{~mm} / \mathrm{s}$. Moreover, the average applied load for all measurements was $1.2 \pm 0.15 \mathrm{~N}$. Friction coefficients were calculated as the ratio of friction force and applied load after removing the turning points

Table 1 The ten different surfaces that were used in the tactile perception study: reference uncoated float glass, and nine types of thin films deposited on float glass

\begin{tabular}{|c|c|c|c|}
\hline Surface type & Coating & & \\
\hline$\# 1$ & Float uncoated & 0 & \\
\hline$\# 2$ & $\mathrm{SiOx}$ & $\bar{\square}$ & \multirow{4}{*}{ "Inorganic" } \\
\hline$\# 3$ & SixNy & $\square$ & \\
\hline$\# 4$ & TiOx & $\square$ & \\
\hline$\# 5$ & $\mathrm{NbOx}$ & $\square$ & \\
\hline$\# 6$ & $\mathrm{SiOx}-\mathrm{CHy}$ & 0 & \multirow{3}{*}{ "Hybrid" } \\
\hline$\# 7$ & $\mathrm{SiOx}-\mathrm{CHy}$ & 0 & \\
\hline$\# 8$ & $\mathrm{SiOx}-\mathrm{CHy}$ & 0 & \\
\hline$\# 9$ & Fluoropolymer I & $\Delta$ & \multirow{2}{*}{ "Organic" } \\
\hline$\# 10$ & Fluoropolymer II & $\Delta$ & \\
\hline
\end{tabular}


associated with changing direction of the finger. The friction force in the "backwards" direction (pulling the finger toward the body) is recorded as a negative number: we present all friction coefficients as the absolute values of the measurements in this paper. One measurement consisted of 15 stroking cycles; however, our observation is that in general perceptual discrimination experiments would be better represented by three strokes since most participants are able to make decisions using less exposure. Thus, the friction data shown are for the first three strokes. In the supporting information, the data for 15 strokes are also shown.

In total, six friction measurements were conducted on each glass sample. Measurements were performed in a climate-controlled room with a temperature of $22{ }^{\circ} \mathrm{C}$ and relative humidity of $45 \%$. One glass surface was used for three friction measurements, where each friction measurement was obtained from a fresh track on the surface. The ten samples were measured in six series in which the order of the ten samples within each series was randomized. The skin hydration was monitored before each series of measurements with a Corneometer CM 825 (Courage + Khazaka $\mathrm{GmbH}$, Germany) and was in the range of 65-70 arbitrary units which is considered a "moist finger."

\section{Physical Characterization}

The water contact angle (WCA) was measured together with contact angle hysteresis, surface free energy (SFE) and the slide-off angle on the freshly coated surfaces. To monitor the surfaces over time, additional control measurements of the WCA were performed at the onset of data collection, and no significant change in WCA over the course of the experiment was observed. The WCA measurements were performed by measuring both sides of a droplet at three different locations on a sample with a Goniometer (Ramé Hart A1100). An average value was then calculated from the six measurements. Roughness/height profile was always defined from $2 \times 2 \mu \mathrm{m}$ zoom-images using an Atomic Force Microscope (Nanosurf Easy Scan) with Al-coated Silicon tip (Tap190AlG, BudgetSensors) at resonance frequency ca. $190 \mathrm{kHz}$ and force constant $48 \mathrm{~N} / \mathrm{m}$. The supporting information section includes the data for WCA, WCA hysteresis and SFE. The latter was obtained from contact angle measurements using three different liquids (diiodomethane, ethylene glycol, water) according to established principles. The WCA was re-evaluated at time of friction measurement to ensure that any surface aging effects would be accounted for.

\subsection{Perception Study}

The participants in the tactile perception study were ten women in the ages of 20-34, (24.8 average age), all right handed. Women were preferentially selected in order to avoid possible convolution of gender differences with the perceptual differences between the surfaces. All procedures were in accordance with the ethical standards of the institutional and the national research committee and with the 1964 Helsinki declaration and its later amendments of ethical standards regarding studies with human participants. Written consent was given, and the participants were informed that they could quit the experiment at any time if they so wished. The only risk for physical injury detected in the risk assessment was the sharp edges of the samples. This was addressed by constructing two sample holders that covered the edges and only exposed the safe area of the samples, see Fig. 1. To run a successful data collection, each sample holder needed to be safe, fast and easy to load, and be able to hold two samples for the paired comparisons. The experimenter handling the samples and loaded/unloaded the sample holders wore protective gloves. The first experimenter loaded the sample holders according to the randomized orders (a unique order for each participant) and the second experimenter presented the samples to the participants and recorded the data.

The participants were instructed to scale the perceived haptic similarity between all possible combinations of pairs of stimuli in one-half of a $10 \times 10$ matrix, i.e., $1-2,1-3, \ldots$ ,1-10; 2-3, 2-4 ... 2-10; .. 9-10. Before the experiment started, the participants washed and dried their hand according to earlier protocols using an unformulated surfactant. After receiving their instructions, they were blindfolded and given a practice session during which they could practice the scaling method and get a feel for the range of materials. The scale for the similarities ranged from 0 to $100 \%$, where $100 \%$ meant that the samples were identical and $0 \%$ as different as possible within the given range. Halfway through

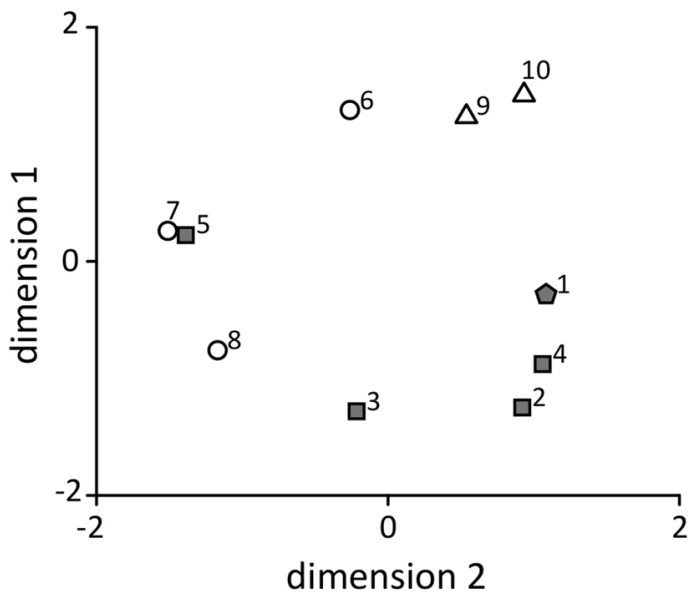

Fig. 1 Two-dimensional tactile space based on perceived similarities among the ten coated glass surfaces. The closer the points in the map, the more similar the coatings are perceived. Point numbers, colors, and symbols are explained in Table 1 
the experiment the participants had a short break, during which they washed and dried their hands again. After the completion of the experiment, the participants were asked to rate the pleasantness of each coating type on a scale from 1 to 10 , where 10 was most pleasant and 1 least pleasant.

The data were analyzed with multidimensional scaling (ALSCAL) using the IBM statistics software SPSS. The scaling of similarity is a relatively unbiased measurement. The scaling was interval data and allowed for individual differences weights to compensate for differences in dimensional preference from the participants, see [10] for further theory and application of multidimensional scaling.

The pleasantness rating is not formally a robust scale and should therefore not be taken too literally. Its purpose was to anchor the dimensionality, i.e., in which direction does a general pleasantness lie, not as an absolute scale. However, the similarity scale can then be consulted for interpreting the relative pleasantness between the samples.

\section{Results and Discussion}

\subsection{Tactile Map}

From the considerable spread in similarity scores across surface pairs (range 19-79), it was clear that our participants were able to detect perceptual differences between the surfaces, see Table 2.

The multidimensional scaling analysis treats the perception similarities as distances and identifies and reveals how many important factors the participants relied on to differentiate between the surfaces. For this data set, the analysis suggested a 2D solution. The stress (0.39) and RSQ (0.16) values for the $2 \mathrm{D}$ solution suggest that although participants were able to successfully perform the task, it was relatively difficult. From the derived subject weights in the scaling analysis, it was also noted that there was variability between participants as to which dimension was primarily responsible for discrimination. The resultant tactile map based on the dissimilarity scores (100-similarity score) is shown in Fig. 1. From the distribution of the samples within the map, it is apparent that the participants perceived the smooth glass surfaces as different. Although this analysis revealed that the participants relied on two perceptual dimensions to discriminate the stimuli, it cannot tell us what these dimensions are. To do this, we attempted to relate the perceptual dimensions to instrumental data.

\subsection{Physical Characterisation}

The average, dynamic finger friction coefficients and standard error of the first three stroking cycles based on six measurements per sample are presented in decreasing order in Fig. 2a. The water contact angle is plotted in Fig. 2b. The uncoated glass has a low contact angle and the fluorocarbon surfaces, as expected, have highest contact angle. Contact angle hysteresis data are plotted in supporting info and is positively related to contact angle. Typically, there is no clear static friction peak in such measurements, so the friction coefficient is obtained from the sliding, or dynamic region of the force traces. Derler et al. [4] have earlier investigated the finger tribology on glass of various roughness and identified adhesive friction as the dominant underlying mechanism. For smooth glass the friction coefficient was around 2.2. Overall, the glass surfaces in this study are also regarded as high friction surfaces with friction coefficients from 2 and above. In comparison, tactile friction coefficients for printing paper lie in the range of 0.2-0.5 [11]. As can be seen, the fluoropolymer-coated glass surfaces (\#9 and \#10) display the lowest friction coefficient and two of the $\mathrm{SiOx}-\mathrm{CHy}$ (\#6 and \#7) display highest friction together with two of the SiOx and SixNy (\#2 and \#3).

Smooth and high-friction surfaces are often associated with a high degree of stick-slip. A detailed look at the load and friction for all measurements show that stick-slip is present on these glass coatings, especially strongly for coatings
Table 2 Mean similarity ratings between the glass coatings

\begin{tabular}{lllllllllll}
\hline & 1 & 2 & 3 & 4 & 5 & 6 & 7 & 8 & 9 & 10 \\
\hline 1 & - & & & & & & & & \\
2 & 63 & - & & & & & & & \\
3 & 70 & 58 & - & & & & & & \\
4 & 65 & 64 & 67 & - & & & & & \\
5 & 79 & 42 & 63 & 41 & - & & & & \\
6 & 60 & 42 & 36 & 67 & 62 & - & & & \\
7 & 60 & 34 & 55 & 66 & 67 & 70 & - & & \\
8 & 75 & 69 & 68 & 58 & 56 & 34 & 74 & - & \\
9 & 74 & 30 & 47 & 26 & 52 & 79 & 56 & 32 & - & \\
10 & 32 & 26 & 19 & 31 & 31 & 51 & 32 & 28 & 55 & - \\
\hline
\end{tabular}

Sample numbers refer to Table 1 


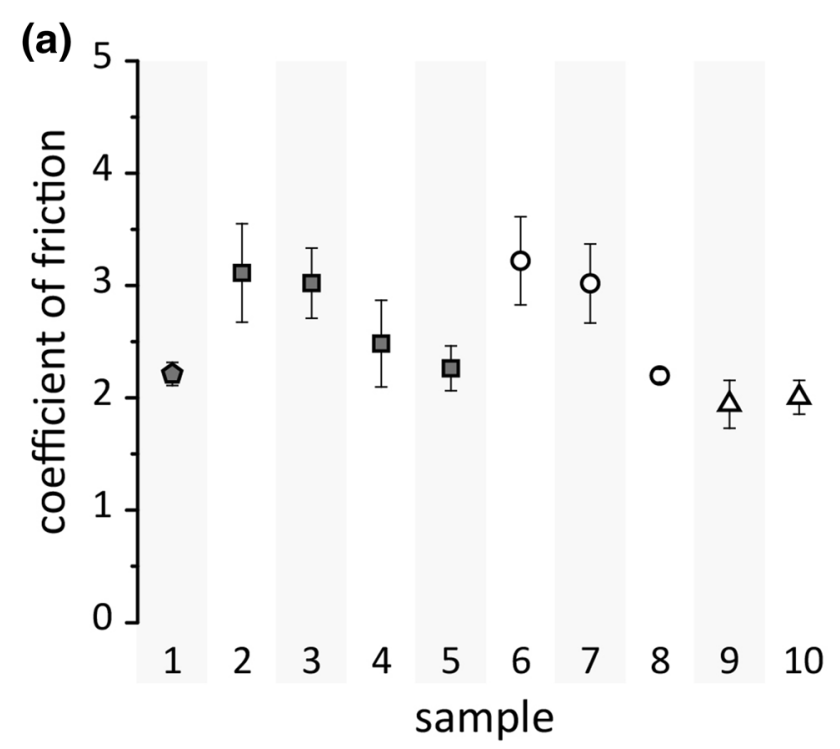

Fig. 2 a Average friction coefficients for the ten different surfaces, calculated from six measurements per surface. The symbols, labels and shading are as described in Table 1 . The error is presented as the

2, 3, 4, 6, 7, and 8. A typical plot of the two forces can be seen in Fig. S1, where obvious stick-slip is "visually" observed during the first few strokes. The disappearance of the stick-slip over time may have several explanations. It could be the result of transfer of lipid material from the fingers that lubricates the surface or, if sufficient moisture is transferred from the finger, this moisture may also act as a lubricant $[4,12]$. Although the finger pad does not have sebaceous glands, there is nonetheless lipid material transfer from the finger to the surface during touch. Similarly, increased moisture in general increases friction due to the softening of the stratum corneum [13] and occlusion [5]. However, because glass is non-absorbent, the accumulation of moisture in the contact also provides a possibility of reducing friction via a lubrication mechanism.

Previous experience $[1,12]$ from tactile friction measurements has shown a friction dependence on the surface roughness where there is an optimum surface structure range or roughness for low friction. This is explained by the real contact area at the finger-surface interface. A surface structure may decrease the real contact area, i.e., smaller number of contact points, compared to a smooth surface, resulting in a lower friction. The glass surfaces within this project are all very smooth, enabling a large contact area between a finger and the surface, resulting in high friction and little expected frictional variation due to roughness differences.

The root mean square (RMS) roughness and average height for the coatings were obtained from AFM images and are listed in Table 3. As can be seen in Fig. S2, the tactile friction coefficient of the coatings does not seem to

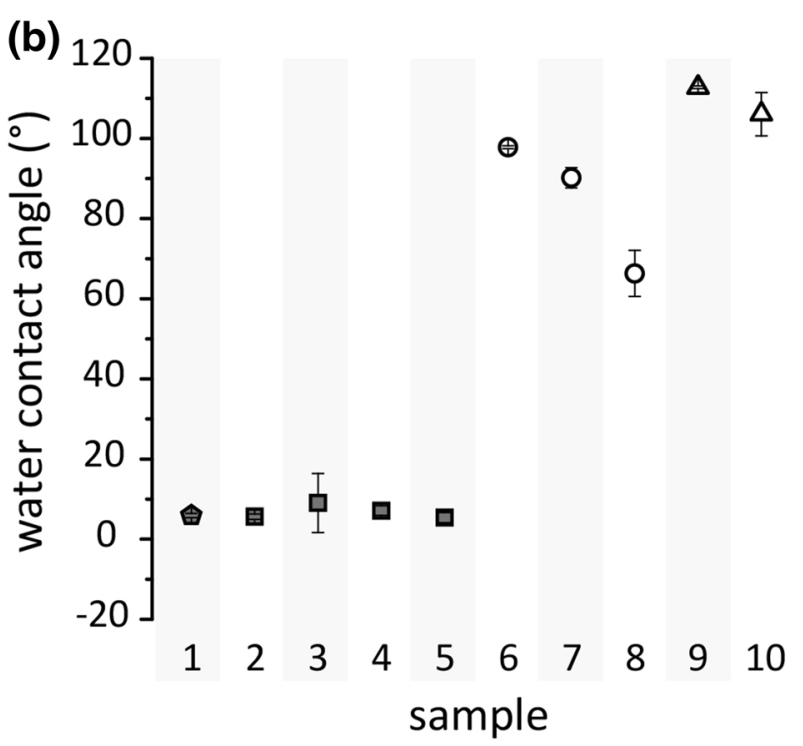

standard error of the mean. b Water contact angles for each of the surfaces. The error is presented as the standard deviation of the measurements

Table 3 RMS roughness and average height for the different coatings obtained with AFM

\begin{tabular}{lll}
\hline Coating & RMS roughness (nm) & $\begin{array}{l}\text { Average } \\
\text { height } \\
(\mathrm{nm})\end{array}$ \\
\hline \#1 Float uncoated & 0.16 & 0.6 \\
\#2 SilOx & 0.25 & 0.9 \\
\#3 SixNy & 1.2 & 3.3 \\
\#4 TiOx & 1.07 & 2.25 \\
\#5 NbOx & 1.8 & 2.57 \\
\#6 SiOx-CHy & 0.62 & 2.14 \\
\#7 SiOx-CHy & 0.39 & 1.37 \\
\#8 SiOx-CHy & 1.28 & 1.3 \\
\#9 Fluoropolymer I & 0.73 & 2.2 \\
\#10 Fluoropolymer II & 0.75 & 1.95 \\
\hline
\end{tabular}

depend on the RMS roughness (nor average height). It is observed that the $\mathrm{SiOx}-\mathrm{CHy}$ coating (\#8) displays higher roughness compared to \#6 and \#7 which may explain why \#8 shows lower friction. However, film \#8 contains the lowest amount of carbon of the samples in the $\mathrm{SiOx}-\mathrm{CHy}$ film family, and within this family a clear trend suggesting that higher C-content is associated with lower friction is evident. AFM images of these three $\mathrm{SiOx}-\mathrm{CHy}$ surfaces are shown in Fig. S3. These samples appear to act as outliers in the tribology behavior and this is discussed further below.

The evaluation of the friction response to differences in surface chemistry is addressed by plotting the friction coefficient versus the water contact angle in Fig. 3. Figure 4 shows 


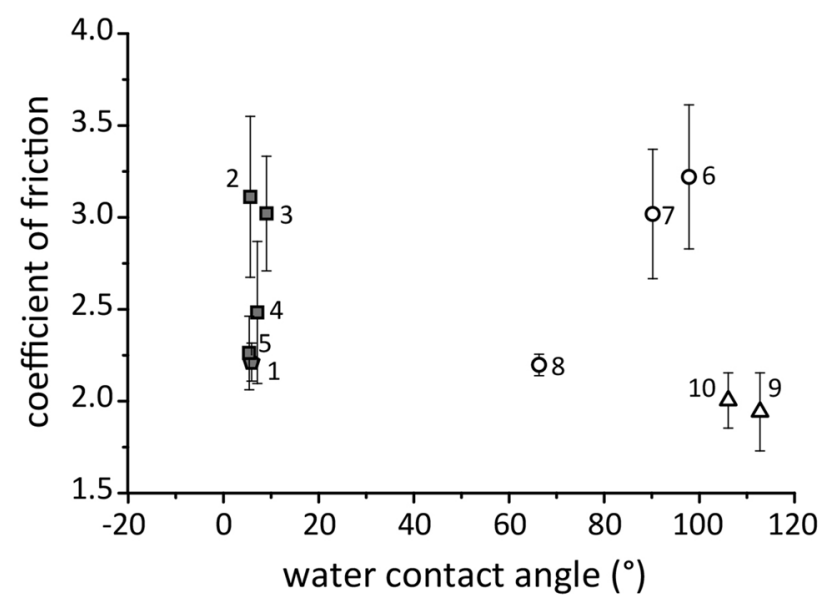

Fig. 3 Tactile friction coefficient versus water contact angle. Point numbers refer to Table 1

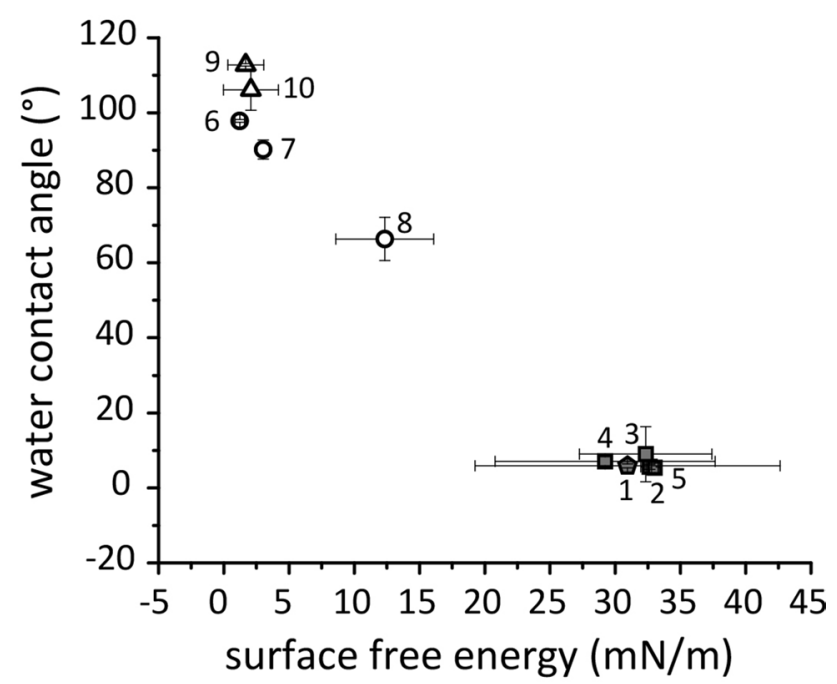

Fig. 4 WCA versus surface free energy. Point numbers refer to Table 1. The strong correlation indicates that the experimental measure (WCA) can be treated as equivalent to the underlying physical parameter it probes - the surface free energy

that the surface energy and the contact angle are inversely related to one another and can therefore regarded as degenerate parameters. As can be seen, the friction is not a systematic function of solely water contact angle (or surface free energy). However, within each sample family, differing behaviors emerged. The two most hydrophobic surfaces (high water contact angle and low surface free energy), the fluoropolymer coatings, produced the lowest friction coefficients. Within the SiOx-CHy family (\#6-8), the friction coefficient increases with increasing water contact angle and decreases with the surface free energy.

Other parameters that may affect the friction response that have not been addressed are the surface charge, and how the moisture in the sliding contact is affected by the surface chemistry of the coating. The differences in friction response between the coatings may be an effect of this, as water contact angle alone was not enough to describe them. The surface free energy and the water contact angle is very similar for the uncoated and the coated glasses \#2-5 as can be seen in Figs. $2 b$ and S4.

The tactile friction measurements in this study are performed in reciprocating strokes, where a forward stroke (away from the body) is recorded as a positive tangential force and a backward stroke as a negative tangential force (towards the body). Analysis of variations in the two stroking directions shows that it is mainly the friction in the backward stroke that varies between the coatings, and that this value tends to be larger (see Fig. 5).

This friction hysteresis has not previously been observed on any of the other materials studied using our apparatus, which include paper [12], artificial skin [14], tissue and polymer. It was observed that on average the applied load in the backward stroke (all measurements) was $1.0 \mathrm{~N}$ while for the forward stroke this value was significantly larger at $1.5 \mathrm{~N}$. When the finger encountered higher friction, a lower load was applied so that the finger could slide on the surface, most probably an unconscious action which has been addressed previously $[12,15]$ and results from the fact that the tendency is to probe with a constant friction force, rather than constant load. A similar hysteresis was observed by

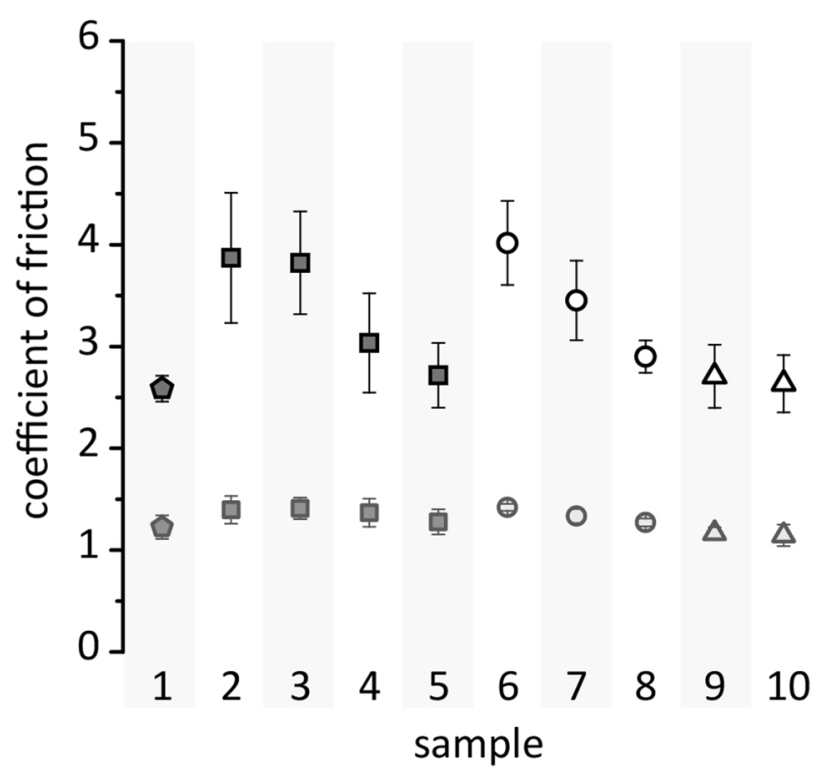

Fig. 5 Friction coefficients of the various samples divided into forward (pushing the finger away from the body, higher friction dark gray and open points) and backward (pulling the finger towards the body, lower friction desaturated points) stroking directions. In this case, the friction coefficients are averaged from all 15 stroking cycles for the 6 measurements per glass sample 
Derler [4] and attributed to mechanical asymmetry. There may also be an additional mechanical contribution to this effect, which is specifically associated with stick slip. Zhang et al. [16] have recently addressed this subject, and conveniently they have done so using glass as a substrate. In this elegant study, they demonstrated that there is a strong hysteretic effect in friction coefficient with sliding direction with a mechanical origin. In fact, the hysteresis depends strongly on the angle of the finger to the surface, and reverses as the angle becomes steeper. At lower sliding angles, analogous to those used here, the friction is higher as the finger is slid towards the body. The authors also measure the spacing of the fingerprint ridges within the nominal area of contact and show that this changes with the sliding direction and thus ascribed the hysteresis to this biomechanical variation. In that study, it was also shown that more reproducible friction data were obtained for an immobilised finger, though this configuration is less representative of the fingers mechanical behavior during actual tactile probing. They also observed changes in load with sliding direction as seen here, so the picture is not fully clear. Because the finger pad is deformable, the friction-load relationship is pseudolinear only over moderate ranges due to the non-linear relationship between nominal contact area and load. Thus, there are likely to be additional hysteretic effects resulting from the variation in load with direction.

Close examination of the extended (15 stroke) friction data indicates that for surface \#6 ( $\mathrm{SiOx}-\mathrm{CHy}$ ), the first three measurements (all performed on the same piece of glass in different tracks) show an average friction coefficient of 3.2, whereas the last three measurements performed on another piece of glass returned a friction coefficient of 4.9. No studies of the coating robustness were undertaken. The water contact angle hysteresis (Fig. S7) may indicate that there are differences in topography in the coating, or that the surface displays patches of hydrophilic and hydrophobic areas. Coating \#6 displays the highest contact angle hysteresis and this may be the reason for the observed variations in friction coefficient-i.e., that friction hysteresis may have a similar advancing/receding mechanistic dependence as that of the contact angle.

\subsection{Correlating Physical and Perceptual Data}

To attempt to interpret the dominant perception dimension, the coordinates from dimension one were plotted against the instrumental measurements. In Fig. 6, the dimension 1 scale values are plotted against the WCA. Although there is not a perfect systematic relationship between the perception and WCA, the relationship is statistically significant, see Table S1. Thus, the analysis suggests that the water contact angle describes dimension 1 well. This implies that the surface chemistry was the main cue used for discrimination in

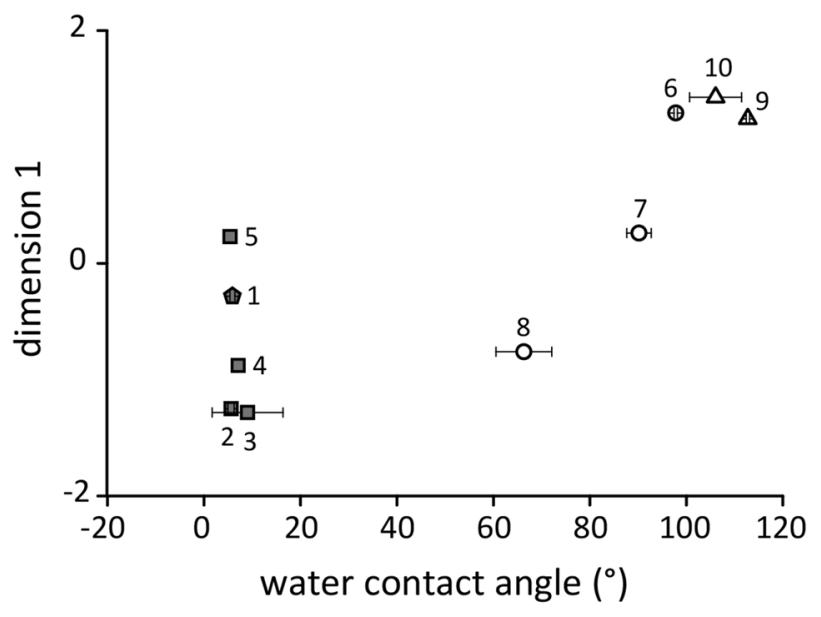

Fig. 6 Dimension 1 scale value versus the water contact angle. Point numbers refer to Table 1

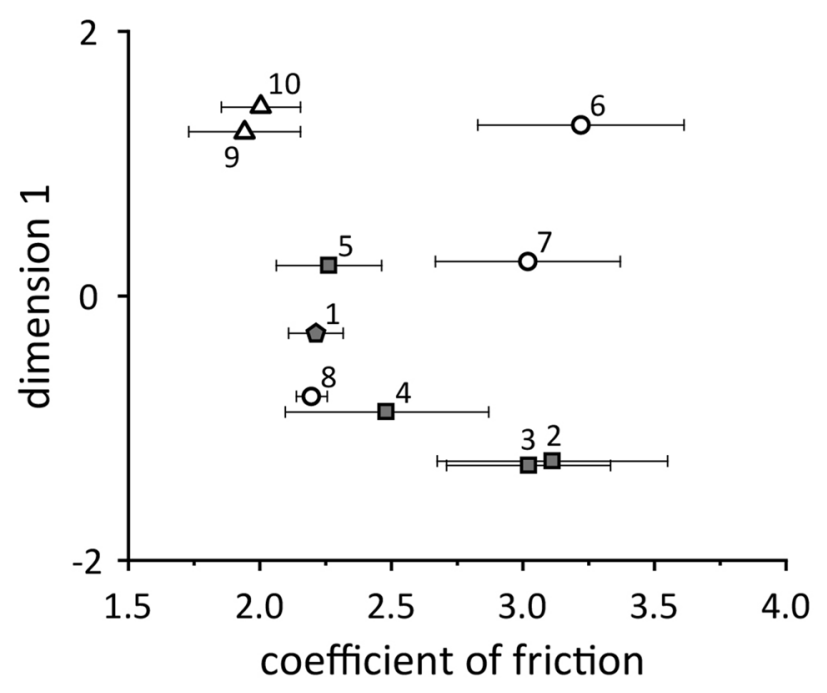

Fig. 7 Dimension 1 scale value versus tactile friction coefficient. Point numbers refer to Table 1

this task. Despite this significant correlation, inspection of Fig. 6 suggests that samples \#1 through to \#5 do not seem to be dependent on the WCA to the same extent as the other samples. We also correlated the coordinates in dimension 1 with the friction coefficient (shown in Fig. 7). Whilst not statistically significant, Fig. 7 suggests that friction contributes to how the participants discriminated the surfaces.

We investigated the possible relative contributions of WCA and friction further by splitting the surfaces into high (\#1-5) and low (\#6-10) hydrophilicity groups. This roughly mirrors the grouping of the coatings into organic and nonorganic. The same correlation analysis was then performed on the two sub-groups. The results are shown in Tables S2 and S3. Interestingly, this analysis implies that for surfaces 
\#6-10, WCA remains the main explanatory parameter for dimension 1, while for surfaces \#1-5, the important factor seems to be the friction coefficient of the surfaces. We interpret this as meaning that for highly hydrophobic surfaces, WCA is the primary cue used for distinguishing surfaces. In contrast, for surfaces with low hydrophobicity, WCA is still a factor however the friction coefficient is more important. This is consistent with the conclusion that surface chemistry is an important cue for tactile perception of surfaces.

While the friction coefficient seems to be important for discrimination in at least some of the samples and explains some of the variance in dimension 1, sample \#6 appears to be something of an exception. As discussed above, there appear to be questions concerning its robustness, and it displays both high contact angle hysteresis and frictional hysteresis. Bearing these reservations in mind a possible explanation for its placement in Fig. 7 is that despite the high friction coefficient, the high WCA was a more dominant feature for the perception of the surface. It thus appears that a finger friction model is suitable for characterizing the perception of glass coatings lower than $100^{\circ} \mathrm{WCA}$, and that for WCAs over $100^{\circ}$ it becomes less meaningful. This is consistent with the analysis for high and low hydrophilic surfaces conducted above. However, there was only one high friction, high WCA sample in this stimuli range so this explanation should be regarded with caution until further research addresses this issue.

The relationship is between dimension 1 and friction is thus not as clear cut as in earlier studies where there was an obvious relationship between friction coefficient and the sticky-slippery dimension for micro textures $[1,18]$. Surface chemistry appears to dominate at high contact angles while friction coefficient becomes more important at low, or very similar, contact angles. Dimension 1 is thus NOT unequivocally a "sticky-slippery" perceptual dimension.

No systematic relationship between dimension 2 and any physical parameters was found, though the samples that displayed most stick slip (Fig. S1) behavior are well spread in this dimension. The sampling rate of the device is too small to allow the frequencies to be reliably extracted but these frequencies appear to be high enough to be detected by Pacinian mechanoreceptors under the skin $[17,18]$. This type of dynamic, "pre-constant slip" has been implicated in perceptual discrimination earlier [7, 19]. It thus seems likely that dimension 2 is related to such an effect, though we have not identified a physical parameter that we can extract from the data to correlate with this dimension. Neither can we rule out that effects involving water occlusion in the sliding contact are responsible for this dimension since the surfaces are both smooth and non-porous/adsorbent [5]. Gueorguiev et al. have studied glass and polymer samples to study the effect of varying surface chemistry on perception. In this case, both the topography and the elastic modulus are varied simultaneously with the surface chemistry, and both these parameters can be used as perceptual discriminators $[1,20]$. Nonetheless, the authors were able to successfully couple related perceptual discrimination to differences in an arbitrary spatial frequency parameter extracted from the tangential force. They thus show very clearly that dynamic effects are implicated, particularly in the so-called partial slip region. This lends further weight to the argument that dynamic events related to slip are responsible for dimension 2.

To our knowledge, there is no study where the surface chemistry has been varied as systematically as done here. While it is relatively easy to obtain hydrophobic monolayers by self-assembly approaches which can be compared to hydrophilic untreated glass on a friction device, it is untenable to use that approach to produce a large number of surface chemistries or to produce the hundreds of samples for each surface chemistry which are necessary for pairwise comparisons using fresh surfaces for every comparison. It should also be noted that while the wetting properties are varied, different chemical approaches are used, which may well have different binding affinities to the glass, or local variations over the large areas of float glass employed. There is no evidence for this, but it cannot be completely ruled out. The use of a single operator in performing friction measurements is not completely without risk. As has earlier been discussed, the variation between individuals can be much larger than the variation between substrates, leading to difficulties in analyzing group data, though the trends are preserved at the individual level [15]. Thus, it should be established that the operator has representative tribological properties, rather than being an outlier, in terms of absolute friction coefficients. Neither is it practical to record friction data on the individual participants-their sessions take several hours for the psychophysical experiments alone.

\subsection{Affective Perception}

Complementary to the similarity data, the ten surfaces were ranked in order from least pleasant (1) to most pleasant (10) by each participant. Note that this is a ranking and not a score, so the abscissa labels of Fig. 8 should not be seen as values, or as representing absolute differences in pleasantness. To assess if assessments of pleasantness were associated with any of the physical parameters, we ran correlations between the mean pleasantness ranking and selected instrumental measurements (Table S4). This analysis revealed a significant correlation between pleasantness and the friction coefficient (COF 3). From Fig. 8, it can be seen that participants ranked \#10 (high contact angle, low friction coefficient) as the most pleasant and \#2,\#3, \& \#7 (high friction coefficients) as the least pleasant. Again, \#6 was a something of an exception. Similar findings relating pleasantness and 


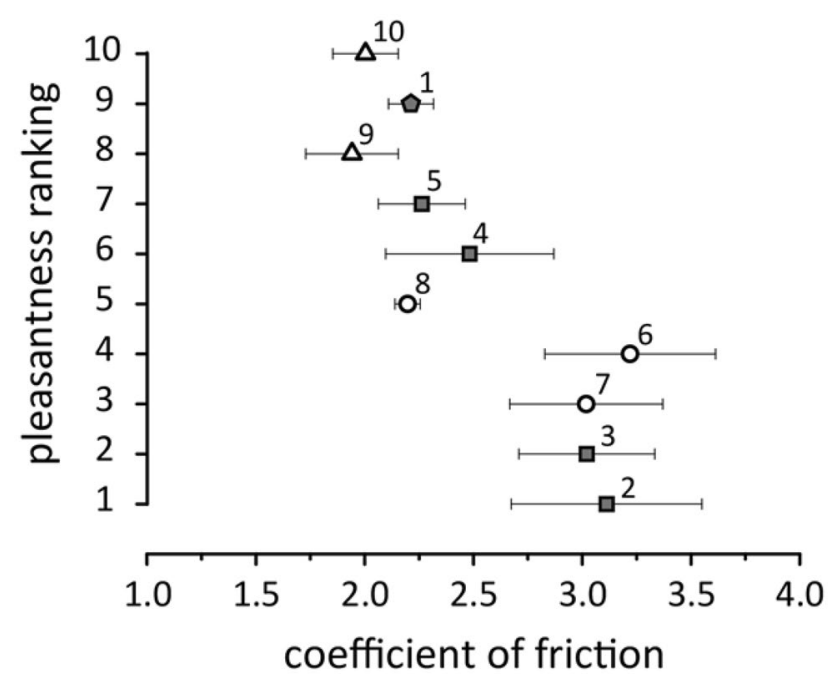

Fig. 8 Pleasantness ranking versus coefficient of friction (3 cycles). Point numbers refer to Table 1

friction coefficient are discussed by Adams et al. [5]. Interestingly, pleasantness was not significantly correlated with WCA or surface energy. This suggests that although WCA was a primary contributor to participants' ability to discriminate between the surfaces, it alone did not affect their pleasantness ratings. Instead, pleasantness is strongly related to the friction coefficient. Together, these findings tend to cement the importance of tribology in affective touch, and suggest that psychotribology is likely to emerge as a distinct field.

\section{Conclusions}

It is clearly possible to use touch to distinguish between identical, smooth materials which vary only in their surface chemistry. The ability to distinguish a molecularly thin film is related to, but not entirely defined by, the friction coefficient, which in turn is related to the true contact area between the finger and surface, as well as local adhesion. Our perceptual dimension 1 is shown to be anchored in surface energy and only partially related to friction and cannot therefore be described as a "sticky-slippery dimension" identified from classical psychophysics. This result thus varies significantly from previous studies where the perceptual dimension appears unambiguously linked to the friction coefficient and is therefore named "sticky-slippery." In fact, in the present work, the surface chemistry, as expressed by the contact angle or surface energy, is primarily responsible for discrimination. As for fine texture perception, two perceptual dimensions appear to be responsible for our ability to discriminate these surfaces. The second perceptual dimension returned from the psychophysical analysis could not be directly correlated to any of the surface chemical data that characterize the systematic stimulus set. This is not uncommon in psychophysics where a perceptual dimension may be linked for example to a combination of parameters. Nonetheless, stick-slip (or vibrational) behavior remains the most likely candidate to explain this dimension particularly in view of recent work described in [7] where analysis of spatial frequencies in the friction data, breakaway friction and partial slip could be correlated with perception. Further work is required to cement this conclusion irrevocably for these flat surfaces but if so, then perception of surface chemistry is analogous to fine texture perception in that it is anchored in two perception dimensions one of which is friction coefficient related, while the other depends on vibrations.

There are very few materials, or product sets, which demonstrate such low roughness as these which is why they are relevant from a scientific perspective-chemistry is isolated from other physical parameters to enable a long-term hierarchical understanding of the mechanisms that underpin touch. Conversely, the general relevance for technology may appear at first sight to be limited. However, the use of chemically altered surfaces to convey a message haptically, for example, the proximity to the edge of a touchscreen, is one possible direct area of application.

Glass in itself has several different areas of application where controlling a tactile signature or function may be important. To apply chemical touch more generally to other materials, where fine or coarse textures are manifested it will be necessary to perform further, more complex studies to observe the hierarchical dependence of the roles of chemistry and texture, as well as to further understand the complex interaction of the finger with the surface. The fact, however, that friction underlies one of the key perceptual dimensions of fine texture, while surface chemistry has a strong influence on smooth touch, implies that texture and surface chemistry can both be applied as tools in attempts to control perception.

Finally, and excitingly, it is also possible to draw conclusions for affective touch. Specifically, our results indicate that while neither WCA or surface free energy alone were associated with pleasantness, the friction coefficient was significantly inversely related to pleasantness. There is thus a clear avenue for affective engineering of products and materials using the frictional properties. While pleasantness is, of course, context dependent, a lower friction coefficient is associated with pleasantness for glass-based materials. Taken together, the results of the present work imply that the emerging new discipline, of which this paper is an example, has a bright future and we suggest the term Psychotribology as a suitable epithet. 
Acknowledgements Mimmi Erikson is thanked for performing the finger friction experiments and Johan Andersson for assistance with handling of the glass samples and the sample holder, both during 2014.

Open Access This article is distributed under the terms of the Creative Commons Attribution 4.0 International License (http://creativeco mmons.org/licenses/by/4.0/), which permits unrestricted use, distribution, and reproduction in any medium, provided you give appropriate credit to the original author(s) and the source, provide a link to the Creative Commons license, and indicate if changes were made.

\section{References}

1. Skedung, L., Arvidsson, M., Chung, J.Y., Stafford, C.M., Berglund, B., Rutland, M.W.: Feeling small: exploring the tactile perception limits. Sci. Rep. (2013). https://doi.org/10.1038/srep0 2617

2. Arvidsson, M.: Getting a Feel for Tactile Space. Stockholm University, Stockholm (2012)

3. Cornuault, P., Carpentier, L., Bueno, M., Cote, J., Monteil, G.: Influence of physico-chemical, mechanical and morphological fingerpad properties on the frictional distinction of sticky/slippery surfaces. J. R. Soc. Interface 12(110), 20150495 (2015)

4. Derler, S., Gerhardt, L.C., Lenz, A., Bertaux, E., Hadad, M.: Friction of human skin against smooth and rough glass as a function of the contact pressure. Tribol. Int. 42(11-12), 1565 (2009)

5. Adams, M.J., Johnson, S.A., Lefèvre, P., Lévesque, V., Hayward, V., André, T., Thonnard, J.-L.: Finger pad friction and its role in grip and touch. J. R. Soc. Interface. 10(80), 20120467 (2013). https://doi.org/10.1098/rsif.2012.0467

6. Pasumarty, S.M., Johnson, S.A., Watson, S.A., Adams, M.J.: Friction of the human finger pad: Influence of moisture, occlusion and velocity. Tribol. Lett. 44(2), 117-137 (2011). https://doi. org/10.1007/s11249-011-9828-0

7. Gueorguiev, D., Bochereau, S., Mouraux, A., Hayward, V., Thonnard, J.-L.: Touch uses frictional cues to discriminate flat materials. Sci. Rep. (2016). https://doi.org/10.1038/srep25553

8. Hollins, M., Faldowski, R., Rao, S., Young, F.: Perceptual dimensions of tactile surface texture: a multidimensional-scaling analysis. Percept. Psychophys. 54(6), 697-705 (1993)
9. Klöcker, A., Arnould, C., Penta, M., Thonnard, J.L.: Rasch-built measure of pleasant touch through active fingertip explorations. Front. Neurorobotics 6, 5 (2012)

10. Borg, I., Groenen, P.: Modern Multidimensional Scaling: Theory and Applications. Springer Series in Statistics. Springer, New York (1997)

11. Skedung, L.: Tactile Perception: Role of Friction and Texture. KTH Royal Institute of Technology, Stockholm (2012)

12. Skedung, L., Danerlov, K., Olofsson, U., Aikala, M., Niemi, K., Kettle, J., Rutland, M.W.: Finger friction measurements on coated and uncoated printing papers. Tribol. Lett. 37(2), 389-399 (2010)

13. Skedung, L., El Rawadi, C., Arvidsson, M., Farcet, C., Luengo, G., Rutland, M.W.: Mechanisms of tactile sensory deterioration amongst the elderly. Sci. Rep. 8, 5303 (2018)

14. Skedung, L., Buraczewska-Norin, I., Dawood, N., Rutland, M.W., Ringstad, L.: Tactile friction of topical formulations. Skin Res. Technol. (2015). https://doi.org/10.1111/srt.12227

15. Skedung, L., Danerlov, K., Olofsson, U., Johannesson, C.M., Aikala, M., Kettle, J., Arvidsson, M., Berglund, B., Rutland, M.W.: Tactile perception: finger friction, surface roughness and perceived coarseness. Tribol. Int. 44(5), 505-512 (2011). https:// doi.org/10.1016/j.triboint.2010.04.010

16. Zhang, M., Mo, J.L., Xu, J.Y., Zhang, X., Wang, D.W., Zhou, Z.R.: The effect of changing fingerprinting directions on finger friction. Tribol. Lett. (2017). https://doi.org/10.1007/s1124 9-017-0843-7

17. Bolanowski, S.J. Jr., Gescheider, G.A., Verrillo, R.T., Checkosky, C.M.: Four channels mediate the mechanical aspects of touch. J. Acoust. Soc. Am. 84(5), 1680-1694 (1988)

18. Weerakkody, N.S., Mahns, D.A., Taylor, J.L., Gandevia, S.C.: Impairment of human proprioception by high-frequency cutaneous vibration. J. Physiol. 581(3), 971-980 (2007). https://doi. org/10.1113/jphysiol.2006.126854

19. Delhaye, B., Lefevre, P., Thonnard, J.L.: Dynamics of fingertip contact during the onset of tangential slip. J. R. Soc. Interface. 11(100), 20140698 (2014). https://doi.org/10.1098/rsif.2014.0698

20. Arvidsson, M., Ringstad, L., Skedung, L., Duvefelt, K., Rutland, M.W.: Feeling fine - the effect of topography and friction on perceived roughness and slipperiness. Biotribology 11(Supplement C), 92-101 (2017). https://doi.org/10.1016/j.biotri.2017.01.002 